\title{
DIMENSIONES LOCALES DE LA SEGURIDAD Y LA COOPERACIÓN TRANSFRONTERIZA EN LA FRONTERA AMAZÓNICA DE BRASIL, Colombia y Perú ${ }^{*}$
}

Viviana García PinZÓN**

\section{Resumen}

Tanto en la visión tradicional de la seguridad como en la perspectiva que enfatiza el papel de las nuevas amenazas, las fronteras ocupan un rol central. Sin embargo, la literatura reciente sobre fronteras ha resaltado las limitaciones para el entendimiento y el estudio de la seguridad y defensa en estos territorios, los cuales siguen siendo poco comprendidos. Por ello, se ha resaltado la necesidad de analizar estos temas teniendo en cuenta las particularidades de dichas áreas. Sin embargo, el problema no se restringe a lo teórico, por el contrario, el proceso de política pública sobre seguridad y cooperación fronteriza en Colombia ha estado controlado por las visiones y los intereses de actores nacionales, siguiendo una dinámica de "arriba hacia abajo" (top-down) que deja poco espacio para la participación de los actores locales. Esto constituye un obstáculo para la territorialización de la intervención pública y su efectividad. Con base en estas consideraciones, el objetivo central de este artículo es describir $\mathrm{y}$ analizar las visiones sobre seguridad, cooperación y relaciones transfronterizas por parte de actores locales en el lado colombiano de la triple frontera amazónica (Brasil-ColombiaPerú). Además de visibilizar las perspectivas de dichos actores, este ejercicio permite evidenciar las debilidades y los desafíos de la política pública de seguridad en áreas de frontera.

\footnotetext{
Este texto hace parte de los resultados del proyecto de investigación "Seguridad y perspectivas de cooperación en la región Amazónica”, del Grupo de Investigación en Seguridad y Defensa, Universidad Nacional de Colombia.

** Estudiante doctoral del Instituto Alemán de Estudios Globales y de Área GIGA y de la Universidad de Marburgo; politóloga, Universidad Nacional de Colombia; magíster en Ciencia Política, Universidad de Chile. Research Fellow; estudiante doctoral del GIGA - Hamburg, (Alemania). [viviana.garciapinzon@giga-hamburg.de].

Recibido: 4 de abril de 2018 / Modificado: 31 de mayo de 2018 / Aceptado: 2 de agosto de 2018.

Para citar este artículo
}

García Pinzón, V. (2018). Dimensiones locales de la seguridad y la cooperación transfronteriza en la frontera amazónica de Brasil, Colombia y Perú. opera, 23, 59-80.

DOI: https://doi.org/10.18601/16578651.n23.05 
Palabras clave: región amazónica, seguridad, territorio, frontera.

\section{LOCAL DIMENSIONS OF SECURITY AND CROSS-BORDER COOPERATION IN THE AMAZON TRI-BORDER AREA OF BRASIL, COLOMBIA, AND PERU}

\section{Abstract}

For both the traditional security perspective as well as the one that emphasizes the importance of the so-called new threats, borders have a central role. However, recent literature on borders has pointed out the weaknesses to grasp security and defense issues in these territories, which remain being poorly understood. In this regard, there is a claim to analyze security in these areas considering borderlands' particularities. Beyond the theoretical debate, these weaknesses have had a real impact in both the process of agenda-setting and policies on security, which in the case of Colombia have followed a top-down approach that reflect the views and interests of national actors, while neglecting the participation of the local ones. Against this background, the main objective of this article is to describe and analyze the visions about security, cooperation and crossborder relationships by local actors on the Colombian side of the Amazonian Triple Border (Brazil-Colombia- Peru). By doing this, the weaknesses and challenges of security policies in borderlands are made apparent.

Key words: Amazon region, security, territory, border.

\section{INTRODUCCIÓN}

La visión internacional y regional respecto a la Amazonia está articulada a través de una narrativa que resalta su importancia estratégica debido a la biodiversidad, su posición geográfica-que abarca ocho países-, y el potencial de desarrollo económico. No obstante, la región también es considerada como un territorio complicado en términos de seguridad; un lugar "peligroso" o "área sin ley" (Trejos, 2015) en el que convergen la precariedad estatal, la presencia del crimen organizado y economías ilícitas, y el deterioro del medio ambiente. Esta doble narrativa ha llevado a que, por un lado, los países miembros de la región la perciban como algo que debe ser protegido, no solo de las amenazas ambientales, sino de la apropiación del territorio por parte de países del norte (Betancourt y Pachón, 2013). Por otro lado, la región es vista como una fuente de problemas para la seguridad nacional; un territorio que en buena parte no ha sido incorporado con éxito al proyecto de Estado-nación y donde tienen presencia actores y dinámicas económicas que desafían el orden estatal (Zárate, 2015).

Esa preocupación por la seguridad no es exclusiva de la región amazónica, por el contrario, en el contexto de la globalización, las áreas de frontera (borderlands) y las prácticas de control fronterizo constituyen un aspecto clave de las políticas territoriales en el siglo XXI (Andreas, 2003). La presencia y el accionar del crimen organizado transnacional y actores armados no estatales en estos espacios han llevado a que sean considerados cruciales en la agenda de seguridad global. Sin embargo, a pesar de su importancia, estos territorios 
siguen siendo poco comprendidos por parte de los actores - nacionales e internacionalesencargados de las políticas sobre el tema (Idler, 2018; Zartman, 2010). Esto se debe, en parte, a las perspectivas convencionales de las Relaciones Internacionales respecto a las fronteras, las cuales se pueden clasificar en dos vertientes: por una parte, la visión globalista que sostiene que en un mundo que cada vez es más interconectado las fronteras se han tornado irrelevantes, dado que los vínculos transfronterizos entre actores sociales y mercados han socavado la primacía de las problemáticas tradicionales de seguridad. En contraste, la concepción realista se enfoca en su carácter militar y la necesidad de defenderlas contra las incursiones militares de otros Estados en nombre de la soberanía nacional. Es decir, una perspectiva que se enfoca en las relaciones interestatales en vez de las transnacionales (Andreas, 2003; Idler, 2018). Ambas visiones se quedan cortas para analizar las problemáticas de seguridad y conflicto de los espacios fronterizos, los cuales presentan un carácter excepcional vis-á-vis la soberanía estatal, la relación entre Estados y las dinámicas de intercambio y movimientos de las poblaciones que la habitan. Es por ello que autores como Idler (2018) plantean la necesidad de analizar las políticas de seguridad y defensa desde el lente y las particularidades de dichas áreas.

Pero las falencias en el abordaje de las fronteras no se limitan a los problemas en las bases teóricas que orientan el debate al respecto, sino que también están relacionadas con la forma como se han conformado la agenda y acción públicas en temas de seguridad y defensa en áreas de frontera. Así, en la mayoría de los casos el proceso de política pública, desde la formación de la agenda hasta la implementación de la decisión (Casar y Maldonado, 2008) ha estado controlado por actores nacionales y ha dejado de lado las visiones y los intereses de los actores locales. El predominio de un patrón arriba-abajo (top-down) en la política pública impide la inclusión de las particularidades territoriales y de las demandas locales en la acción pública y, por consiguiente, la respuesta efectiva a la problemática socialmente relevante (Vargas, 2011). Dado el carácter interméstico de los problemas de seguridad en las fronteras, la cooperación es un elemento clave en la política pública. Sin embargo, en esta se replica la misma falencia, de manera que usualmente la acción pública está coordinada desde las capitales de los respectivos países sin tener en cuenta a los actores que conforman y viven en el territorio.

Con base en el debate teórico sobre fronteras y seguridad, así como en la necesidad de posicionar al territorio como elemento clave en el proceso de política pública, el presente artículo desarrolla un análisis sobre la seguridad y las relaciones transfronterizas en el caso de la triple frontera amazónica (Brasil-PerúColombia) a partir de la perspectiva de actores locales (Estado y sociedad civil) del lado colombiano. Según Boisier (2009), las triples fronteras han adquirido renovada importancia en el periodo reciente, debido a que se las considera zonas geoestratégicas claves por los fenómenos de migración ilegal, contrabando, narcotráfico, pero también por el comercio transfronterizo, la diplomacia y la glocalización. Para el caso específico de Colombia, los espacios de frontera se han caracterizado por 
la precariedad de la presencia estatal (Zárate, 2015 , 2017), la marginalidad de las poblaciones de estos territorios y su exclusión de los procesos de decisión en materia de política pública; así como una narrativa desde del centro que concibe estos territorios como peligrosos y fuente de amenazas para la seguridad nacional (Gallo, 2014). En este sentido, un análisis como el que se propone contribuye, desde una perspectiva poco explorada en la literatura nacional, al estudio de lo que hasta ahora ha sido un territorio incomprendido (Zárate, 2915, p. 76). Metodológicamente, el análisis se basa en información primaria y fuentes secundarias. El principal insumo de información primaria lo constituyen entrevistas semiestructuradas con funcionarios y miembros de la sociedad civil realizadas en los municipios de Leticia y Puerto Narińo (Colombia).

El texto se compone de dos partes: en la primera se desarrolla una breve presentación del marco teórico y conceptual sobre territorios fronterizos y seguridad. En la segunda se aborda concretamente el caso de estudio. En este apartado, tras la presentación de generalidades sobre la región, se analiza la agenda de seguridad desde la mirada estatal central y, posteriormente, se profundiza en la escala local.

\section{ÁREAS DE FRONTERA Y SEGURIDAD}

La noción de frontera ha sido utilizada para denominar realidades fácticas, metafóricas o imaginarias construidas social o disciplinariamente, se trata de un "concepto polisémico al que se recurre con distintas acepciones dentro de las ciencias sociales"
(Bartolomé, 2005). No obstante, la mirada tradicional sobre las fronteras estatales remite a la paz de Westfalia (1648), a partir de la cual se sentó una visión que concibe a las fronteras como demarcaciones de los Estados nacionales y de las sociedades que los habitan (Tapia, 2012, p. 179). Al respecto, Gottman (1973 citado por Machado et al., 2009, p. 99) señala que en geografía política una frontera internacional es considerada como una zona de indeterminada anchura cuya característica fundamental es que está cruzada por una línea que determina los límites de la soberanía territorial entre dos Estados. Siguiendo esta definición clásica, se encuentra que el estudio de las fronteras ha estado asociado a las líneas que separan la soberanía de los Estados en el sistema internacional (Newman, 2003); esta es una visión geopolítica de la frontera como elemento de demarcación de la soberanía de un Estado, que ha llevado a que en gran parte de la literatura, límite territorial y frontera sean tratados como sinónimos.

Sin embargo, hay una diferencia entre los límites fronterizos y las áreas de frontera: el límite se concibe como la constitución de líneas de separación físicas y visibles entre espacios económicos, políticos y sociales. Entre tanto, el área de frontera es la región o área en relativa proximidad al límite, donde las dinámicas de cambio y las prácticas cotidianas están afectadas por la misma presencia del límite (Newman, 2006). Como bien lo señala Lía Machado (2000), mientras los límites fronterizos son obra de los Estados que a través de instrumentos jurídicos marcan la separación entre unidades políticas soberanas y delimitan el territorio bajo control estatal; las 
áreas de frontera son construcciones sociales de los pueblos, los cuales se pueden expandir más allá del límite jurídico del Estado, desafiar la ley territorial de cada Estado limítrofe y, a veces, crear una situación de facto potencialmente conflictiva. Las dinámicas y fronteras culturales, económicas y sociales no siempre coinciden con los límites estatales; de manera tal que las áreas de frontera son espacios de movimiento, intercambio e integración en variadas formas, cuyo desarrollo territorial se da en un marco que va más allá de la división político-administrativa de los Estados, e integra actores subnacionales y actores no estatales. Así, las fronteras son una construcción socioterritorial producto de los discursos jurídicos y geopolíticos de los Estados, por una parte, y de la acción de las comunidades fronterizas, por otra.

En el debate de las relaciones internacionales es posible diferenciar dos vertientes de análisis respecto a las fronteras. Por un lado, la perspectiva globalista que sostiene que, dado el incremento y la aceleración de los intercambios de bienes, servicios e información, y la interacción entre diferentes actores que caracteriza la globalización, las fronteras tienen un papel cada vez más marginal en la medida en que se hacen difusas y abiertas. Por otro lado, la corriente realista enfatiza el carácter militar y geopolítico de las fronteras. Dado que la mayoría de las guerras interestatales han sido por la conquista del territorio, el pensamiento geopolítico ha enfatizado la centralidad de la competencia y la adquisición del territorio en las dinámicas de la política mundial y la función militar de las fronteras. Empero, el declive de las guerras interestatales y las transformaciones en la política mundial han evidenciado la debilidad de este enfoque para la comprensión de las relaciones y nuevos fenómenos en el sistema internacional.

Ambas perspectivas presentan limitaciones para entender las dinámicas de seguridad en las áreas fronterizas. Respecto a la visión globalista, si bien es cierto que el control fronterizo se ha hecho más flexible, ello no implica la emergencia de un mundo sin fronteras o la retirada del Estado de dichos territorios. Todo lo contrario, la administración de las fronteras está imbricada en la definición contemporánea del Estado-nación y, a pesar de la pérdida de soberanía estatal en campos tales como la economía, el Estado sigue reclamando una posición central en el ordenamiento de flujos dentro y fuera de los límites nacionales.

El control sobre las poblaciones fronterizas parece haberse fortalecido, tanto en relación con la circulación de personas como de pequeñas mercaderías del llamado "contrabando hormiga". Así, en muchos casos, los pobladores fronterizos perciben una mayor - no una menor-presencia estatal. El Estado se retira de su función de protección y reaparece en su papel de control y regulación. En otras palabras, podríamos estar asistiendo, más que una desterritorialización generalizada, a la sustitución de un modelo de territorialización por otro (Grimson, 2004, p. 11).

Sin embargo, estas formas de control, y las amenazas que el Estado considera, distan de seguir un modelo tradicional como el que plantea la corriente realista. Así, autores como Andreas (2003) y Grimson (2004) han señalado la emergencia de un nuevo modelo de territorialización que trae consigo una va- 
riación histórica en las prioridades de control fronterizo. A medida que las fronteras se hacen cada vez más porosas por efectos de la globalización, los Estados han construido barreras contra el flujo de elementos "indeseables", contra la ilegalidad, o contra los "actores transnacionales clandestinos" (Andreas, 2003). Por ello, Grimson (2004) señala que:

... el Estado no se ha retirado completamente, sino que ha cambiado su eje de intervención. Si en la fase anterior su obsesión era la preservación territorial, el control del espacio, ahora su eje de acción se vincula a controlar los flujos, los movimientos de personas y mercaderías entre los países. Especialmente a promover los "flujos por arriba” y controlar los flujos por "abajo" (p. 12).

Aguiar (2010) habla del neoliberalismo selectivo para dar cuenta de esta dinámica; así, mientras el neoliberalismo promueve una ideología de libre mercado y la reducción del control del Estado, también se construyen nuevas barreras.

Pero no es solo la variación en las formas de control territorial por parte del Estado lo que determina la particularidad de las zonas fronterizas. Las características de la presencia estatal, la relación del Estado con las sociedades en las áreas de frontera, así como la relación entre las comunidades transfronterizas son aspectos fundamentales. En este sentido, parte de la naturaleza de las áreas de frontera es su carácter transnacional y la distancia de los centros políticos y económicos (Idler, 2018, p. 6). Esa distancia configura una lógica de centro-periferia entre las áreas de fronteras y las respectivas sociedades nacionales; esta lógica atraviesa también al Estado (Zartman, 2010). Es decir, en vez de una entidad monolítica, el Estado está moldeado por ese conjunto de relaciones entre centro y periferia que se traducen en una presencia desigual a lo largo del territorio, por un lado, y en una diferenciación entre el Estado nacional (central) y su expresión territorial a escala local (periferia), por el otro. Estos aspectos conllevan usualmente, que no en todos los casos, una débil gobernanza estatal $^{1}$ (Idler, 2018, pp. 6, 7), donde la precariedad del Estado se combina con la existencia de actores no estatales que ejercen funciones de control territorial y social. Dicha situación no implica necesariamente una relación de conflicto u oposición, por el contrario, en muchos territorios fronterizos esta dinámica se encuentra normalizada como parte del orden social (López y Tuesta, 2015).

En contraste con los débiles lazos entre las sociedades de las áreas de frontera y los respectivos Estados, las dinámicas sociales de sus habitantes rebasan las múltiples fronteras políticas de la región (Vargas, 2010; Aponte, 2011). Como Zartman (2010) acertadamente señala, el movimiento y las interacciones locales es lo que caracteriza a las áreas de frontera, lo que las convierte en un espacio excepcional. Esa excepcionalidad afecta las relaciones entre los Estados a escala nacional (central) y a escala local (periferia); por ello, su interacción en el ámbito diplomático y desde la perspectiva nacional, usualmente controlada desde las

\footnotetext{
Cabe señalar que las fronteras también pueden tener un control férreo por una autoridad central fuerte. La frontera entre las dos Coreas es un ejemplo de ello (Zartman, 2010, p. 6).
} 
capitales, no es la misma de los Estados en el territorio. Teniendo en cuenta que la implementación de política pública a nivel local está constituida por el entramado estatal conformado por el Gobierno central y los Gobiernos locales (Vargas, 2011), la territorialización de la intervención pública pasa por considerar las particularidades institucionales que caracterizan a las áreas de frontera.

\section{TRIPLE FRONTERA AMAZÓNICA: CARACTERÍSTICAS GENERALES}

Las áreas de frontera de Brasil, Perú y Colombia conforman una región transfronteriza en la región amazónica (Zárate, 2017); en ella, el vértice de los límites entre los tres países conforma el lugar conocido como Trapecio Amazónico, el cual abarca la porción de territorio colombiano entre los ríos Putumayo y Amazonas, el área fronteriza colindante que incluye las poblaciones brasileńas de Tabatinga, Benjamin Constant y Atalaia do Norte, y las peruanas de Santa Rosa e Islandia (Mapa 1). De importancia crucial en la región es el eje urbano Leticia (Colombia)-Tabatinga (Brasil), y el centro poblado de Santa Rosa en el lado peruano. El Trapecio es el resultado de la negociación política de las fronteras entre los tres países, en la que Colombia logró asegurar su acceso al río (Aponte, 2007). Entre estos, se destacan el Tratado de Navegación y Límites de 1851 entre Brasil y Perú, que creó la línea Tabatinga-Apaporis, la firma del Tratado Salomón Lozano en 1922 entre Colombia y Perú, y la guerra colombo-peruana entre 1928 y 1932.

A finales del siglo xIx, la configuración de una economía de enclave basada en la explotación del caucho conectó a la región con el mercado mundial. La extracción cauchera jalonó la presencia de los incipientes Estados republicanos en la zona, particularmente en los casos de Perú y Brasil. Colombia se incorporó a esta dinámica de forma tardía, afectada por la amplia explotación peruana del caucho que se extendió dentro de su territorio. Aponte (2007) señala que para Brasil y Perú el río Amazonas ha sido central en su construcción regional y nacional, no así para Colombia, donde la construcción territorial como Estado ha se ha concentrado en espacio andino.

Entre las ciudades de Leticia y Tabatinga hay una complementariedad espontánea en actividades económicas y sociales que conforma el núcleo económico, poblacional y político-administrativo de la zona ${ }^{2}$. En el contexto territorial amazónico, estas ciudades son

... reflejo del complejo proceso de fronterización entre Brasil, Colombia y Perú en la Amazonia, generando un área de triple frontera sobre el río Amazonas. Las dos ciudades no nacieron juntas: se encontraron en el límite como resultado del crecimiento económico y demográfico de la década de 1980, que aceleró el enlace entre ellas, configurando hoy un continuo urbano transfronterizo (Aponte, 2011, p. 202).

2 Al respecto, autores como Vergel (2008) han caracterizado a estas como "ciudades gemelas", concepto frente al cual Aponte (2011 y 2016) ha sido crítico, al señalar que más que una dinámica de "espejo” en las ciudades lo que se encuentra en el caso de Leticia y Tabatinga es un complejo urbano transfronterizo. 
MAPA 1. TRIPLE FRONTERA AMAZÓNICA

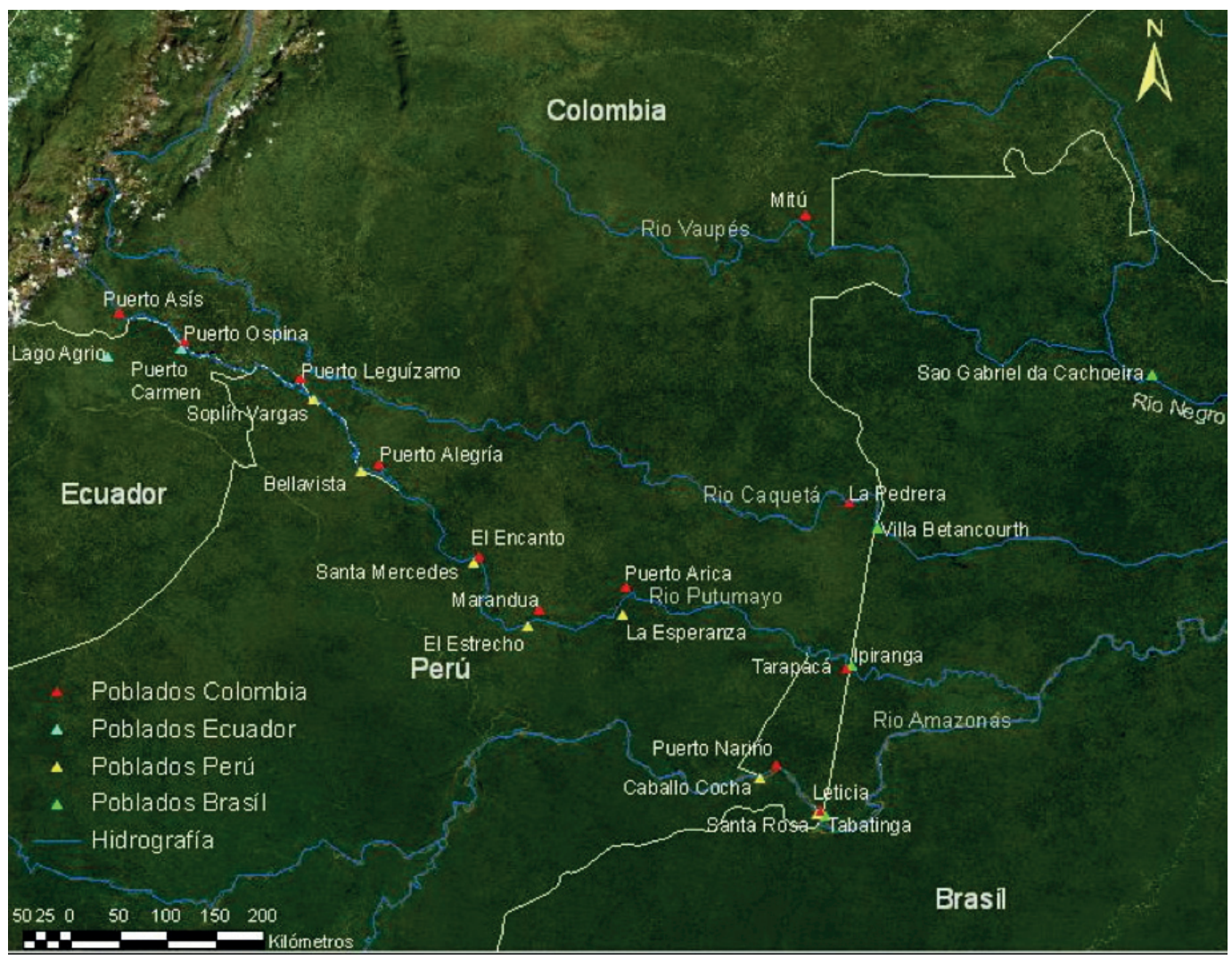

Fuente: Zárate y Aponte (2016).

Este eje urbano es un punto de articulación de los flujos y las relaciones de las áreas pobladas de menor tamaño distribuidas a lo largo de la cuenca hidrográfica del Amazonas. Si bien el intercambio entre los centros poblados de la cuenca es bastante intenso, se trata de una región aislada, con serias dificultades de acceso. Llegar al centro político y económico más cercano en el lado de Brasil, que es Manaos, toma de 6 a 8 días por vía fluvial, con una distancia de $620 \mathrm{~km}$. La parte peruana, Iquitos, se encuentra a una distancia de $370 \mathrm{~km}$ de Santa Rosa y 325 km de Caballo cocha, lo que representa tres días de viaje en barco. A su vez, para ir desde Iquitos hasta Lima, la única opción es la vía aérea con una distancia de $1.010 \mathrm{~km}$. Finalmente, en Colombia, entre Leticia y Bogotá no hay carretera, por lo que solo se puede llegar en avión (distancia de $1.100 \mathrm{~km}$ ) o tras varios días de viaje por río desde Puerto Leguízamo, navegando por el río Putumayo hasta el Amazonas. No obstante, vale señalar que, si bien la Amazonia es una región periférica para el Estado colombiano, 
para el caso concreto de la triple frontera hay un proceso de territorialización diferente. Dado que Leticia es la capital del departamento de Amazonas, la presencia institucional es mucho mayor que en el resto de la región. Como veremos en el siguiente apartado, esta condición tiene consecuencias en la forma en que el Estado opera en este territorio y la relación con los demás actores estatales y no estatales. Eso ha hecho que se replique una relación centro-periferia de Leticia respecto a los centros poblados colombianos cercanos (Aponte, 2011).

A pesar de su condición periférica, como parte de la Amazonia, la triple frontera es un territorio de importancia estratégica que se encuentra enclavado en una zona con corredores biológicos ricos en recursos naturales, en torno a los cuales se están construyendo megaproyectos de infraestructura vial, energéticos, extractivos y agropecuarios. Entre sus características actuales se encuentra un notable crecimiento demográfico, especialmente de poblaciones urbanas (Simmons et al., 2007). El anillo de población en el Amazonas ya no puede ser considerado solo como área de frontera, toda vez que hay economías estructuradas que no están circunscritas al proceso de expansión propio del proceso de poblamiento de la región (Aragón, 2007, p. 163). Dicho crecimiento ha tenido como correlato la desigualdad social y la presencia de conflictos entre los pobladores, algunos de vieja data, tales como los conflictos por tierras, y otros más recientes debido a la presencia de nuevos actores en la zona y al involucramiento de la Amazonia en redes comerciales legales e ilegales de escala local, nacional e internacional (Aponte, 2011; Simmons et al., 2007). Aunque la región constituye un enclave en medio de la selva, con débiles lazos con sus respectivas sociedades nacionales, el avanzar en su comprensión implica dejar de lado aquellas visiones del Amazonas como vacío demográfico y el patrón de desarrollo a partir del imperativo del uso predatorio de sus riquezas naturales, lo que Becker denomina patrón de economía de frontera (Becker, 2005, p. 72), entendiendo que no es solamente un espacio nacional-aunque la dinámica interna de cada uno de los países sean un factor de importancia clave-, sino una región en sí, lo que Zárate (2017) denomina una región transfronteriza.

\section{AGENDA DE SEGURIDAD EN LA TRIPLE FRONTERA: PERSPECTIVAS NACIONALES}

En este apartado se presentan de manera general los aspectos que conforman la agenda de seguridad nacional respecto a la región por parte de cada Estado que conforma la Triple Frontera ${ }^{3}$, a fin de brindar elementos de estudio y contraste respecto a la visión de los agentes estatales y civiles a nivel local, los cuales serán abordados en el siguiente acápite.

Las problemáticas de seguridad en la triple frontera son el resultado de procesos espaciales multiescalares, donde lo local y regional interactúan con actores y fenómenos globales (Simmons et al., 2007). En el marco de una agenda más tradicional de seguridad

3 Cabe señalar que el análisis detallado de los planes y proyectos excede el objetivo y espacio de este artículo. 
centrada en la soberanía estatal, la injerencia de actores externos -sobre todo países del norte- en el marco de la internacionalización de la Amazonia constituye una amenaza para los países de la región, en particular para Brasil (Veyrunes, 2008). Desde la seguridad ambiental, las actividades extractivas y de explotación de recursos naturales, la deforestación, la agricultura extensiva y la expansión urbana (Vergel, 2008) son amenazas para la riqueza natural y la biodiversidad de esta región. En tercer lugar, las economías ilícitas y el crimen organizado (López y Tuesta, 2015; Pabón, 2012; Trejos, 2015; Veyrunes, 2008), donde el narcotráfico es la problemática más protuberante, en este sentido:

.. la importancia del Amazonas para el narcotráfico radica en todas las vías fluviales con las que cuenta, ya que muchas directa o indirectamente desembocan en el río Amazonas en Brasil y de ahí salen al resto del mundo (especialmente Europa): a través del Océano Atlántico la cocaína llega hasta algunos países insulares de África Occidental, antiguas colonias portuguesas, tales como las Islas de Cabo Verde y Guinea Bissau y su archipiélago de los Bissagos. Es importante destacar que los insumos para el procesamiento de la coca llegan desde Brasil, en cuyo territorio también se encuentran laboratorios de cristalización y puntos de comercialización (Trejos, 2015).

Finalmente, desde la seguridad humana, los pobladores de la región carecen de garantías para su bienestar social y económico. La po- breza, la falta de acceso a servicios e instituciones, y a la tierra necesaria para la subsistencia hacen parte de las problemáticas que afectan a las comunidades de la región (Simmons et al., 2007; Vergel, 2008).

La naturaleza interméstica de las amenazas a la seguridad ha llevado al desarrollo de diversos acuerdos e instrumentos de cooperación para la gestión conjunta de las políticas de seguridad (Vargas, 2010). En este sentido, la Organización de Cooperación Amazónica (отCA) ha sido un espacio donde los Estados han discutido la necesidad de abordar las problemáticas de seguridad de manera conjunta ${ }^{4}$ y han proyectado un instrumento con este propósito. No obstante, son los mecanismos de cooperación transfronteriza de carácter multi y bilateral los que conforman la base de la cooperación en este sentido; estos abarcan desde zonas de integración fronteriza, hasta acuerdos de cooperación para la lucha contra el narcotráfico, intercambio de información policial y de inteligencia, y compatibilización de las legislaciones nacionales. Poner en práctica los acuerdos de cooperación no es una tarea fácil, no solo porque involucra la participación de diferentes escalas de los gobiernos (nacionales, estatal-departamental y municipal), y por la falta de recursos y capacidades institucionales en territorios históricamente caracterizados por una débil presencia estatal (Hurtado y Aponte, 2017), sino por la existencia de órdenes sociales locales constituidos por la interacción de actores estatales y no

\footnotetext{
4 Al respecto ver las siguientes declaraciones de las Reuniones de Ministros de Relaciones Exteriores de la Orga-
} nización del Tratado de Cooperación Amazónica (отсA): Caracas (2000), Manaos (2004) e Iquitos (2005). 
estatales, economías ilícitas, e instituciones informales ${ }^{5}$. Considerar estos aspectos nos lleva a un análisis que va más allá de la diplomacia y la cooperación coordinada por funcionarios nacionales desde las respectivas capitales, para adentrarse en las dimensiones locales de la (in) seguridad y la gobernanza. A ello se suma el hecho de que cada país tiene visiones y prioridades distintas respecto a las problemáticas de seguridad de interés. Estas diferencias, y el hecho de que, para los países, a excepción de Brasil, la Amazonia no es prioritaria, constituye uno de los mayores obstáculos para la articulación de esfuerzos entre los países parte de la frontera que dé lugar a esquemas de seguridad cooperativa.

En este sentido, las iniciativas de mayor impacto en la seguridad de la zona fronteriza han sido llevadas a cabo por Brasil. Para este país, la Amazonia constituye un lugar para ser protegido (en términos ambientales y de seguridad), y una zona estratégica para su inserción global y sus intereses como líder regional (Londoño, 2015). Así, "el Amazonas es el tema más sensible y estratégico en el desarrollo de la agenda de seguridad brasilera, proporcional a que es percibido como una amenaza a los intereses tradicionales de Brasil" (Bitencourt, 2002 , p. 81). Frente a una agenda de seguridad que contempla amenazas tradicionales y nuevas amenazas, Brasil ha respondido con iniciativas estratégicas que han sido implantadas desde mitad de la década de los ochenta, a saber: el Programa Calha Norte, el Sistema para la Protección de la Amazonia (SIPAM) con su brazo operativo, el Sistema de Vigilancia de la Amazonia (SIVAM) y el fortalecimiento de la presencia militar en la frontera norte (Guedes da Costa, 2002; Londoño, 2015; Martins y Zicker, 2000). Un ejemplo de lo último es el vasto plan de presencia militar con el propósito de combatir el tráfico ilícito de drogas, el contrabando de armas y municiones, la extracción ilegal de minerales y maderas, el tráfico de animales silvestres y la biopiratería implementado durante el gobierno de la expresidenta Dilma Rousseff. La Operación Ágata 1 tuvo como lugar las poblaciones de Tabatinga y São Gabriel de Cachoeira. En ella participaron más de 3000 militares que contaron con el apoyo de la Policía Federal, la Agencia Brasilera de Inteligencia (ABIN) y el Instituto Brasilero del Medio Ambiente y Recursos Naturales Renovables (IBAMA), entre otros.

Para Perú, la problemática de mayor interés en la agenda de seguridad en la triple frontera, y en particular en el Trapecio Amazónico, es el narcotráfico. Si bien la frontera había sido considerada tradicionalmente co-

5 Entendiendo a las instituciones como "normas, recursos o símbolos institucionales, procesos de políticas, códigos, cultura y comunicación orientan el comportamiento de los actores” (Mariscal, 2003, p. 36), el nuevo institucionalismo señala la existencia de instituciones formales e informales. De manera general, las instituciones informales son aquellas que no están escritas o codificadas. Estas se caracterizan por su opacidad y por ser identificables en las prácticas y opiniones. De manera que, "[e]n la institucionalidad informal el orden de la regla es garantizado por tradición, en el sentido de que es práctica regular, tácita, y recíprocamente aceptada y autosancionada, susceptible al particularismo" (Navarrete, 2017, p. 287). 
mo una zona de tránsito de cocaína, durante los últimos años ha crecido el cultivo de coca en las poblaciones peruanas que hacen parte de la cuenca hidrográfica y que integran la zona fronteriza. Según cifras de Naciones Unidas entre 2001 y 2016 la superficie de cultivo de coca en los valles de Marańon, Putumayo y Amazonas ha aumentado, pasando de 1.250 a 3.383 hectáreas (UNODC, 2017). Caballacocha, Cuchillococa y Bellavista son consideradas como las poblaciones con mayor índice de cultivo de coca en la zona de la triple frontera. El Gobierno peruano ha intervenido en este territorio a través del Programa de Apoyo al Desarrollo Sostenible en la Zona Fronteriza (Caballo cocha, Cushillococha y Palo Seco). El programa surge de la articulación de la Estrategia Nacional de Lucha contra las Drogas y la Estrategia Nacional de Desarrollo e Integración Fronteriza; este no solo se plantea como una estrategia de erradicación de drogas, sino como un esfuerzo de construcción de Estado en un territorio que históricamente ha sido marginado. En este sentido, vale señalar que una de las diferencias que se evidencia en la triple frontera es que mientras Leticia (Colombia) y Tabatinga (Brasil) constituyen ciudades con un dinamismo social y económico, el centro poblado peruano más cercano es Santa Rosa de Yavarí, una pequeña localidad con apenas 2.500 habitantes que hace parte de la Provincia de Mariscal Ramón Castilla en el departamento de Loreto. Teniendo en cuenta la marcada asimetría, este programa tiene como objetivo central la creación de una ciudad que pueda integrarse a la dinámica urbana transfronteriza generada por Leticia y Tabatinga.

En el caso de Colombia, la principal preocupación la constituye la presencia de ac- tores armados ilegales en la región amazónica. Por sus características, los territorios fronterizos han servido como retaguardia de los grupos guerrilleros y corredores estratégicos para los tráficos ilegales que sirven como fuente de financiación para dichos grupos. La trayectoria de construcción del Estado colombiano, al igual que otros países de la región, ha priorizado las zonas centrales en desmedro de los territorios de frontera. El Estado colombiano se ha caracterizado por la precariedad en el despliegue del poder infraestructural a lo largo de todo el territorio. Un análisis de los patrones de ocupación territorial devela que el Estado ha tenido un ritmo de expansión territorial más lento que el de los pobladores, de manera que en buena parte del territorio nacional ha estado ausente o ha tenido que compartir su poder con otros actores, de manera que no ha logrado constituirse en el referente para la resolución de conflictos.

En el año 2014, el Departamento Nacional de Planeación (DNP) publicó el CONPES 3805 de 2014, "Prosperidad para las fronteras de Colombia”, documento que establece el marco para la política de fronteras colombiana. El texto parte por reconocer la condición de marginalidad de los territorios fronterizos respecto al resto del territorio nacional y las considerables brechas socioeconómicas. En el caso del departamento del Amazonas, donde se encuentra el Trapecio Amazónico, el diagnóstico señala, entre otros, los siguientes problemas: actividades ilícitas transnacionales; baja capacidad institucional pública para el aprovechamiento, la conservación y la protección de recursos naturales; deficiente formación de capital humano en los territorios fronterizos; aislamiento y débil control territorial, e 
insuficientes opciones de transporte aéreo de personas y productos, y de infraestructura aeroportuaria, lo cual afecta las potencialidades comerciales y económicas.

\section{VISIONES LOCALES SOBRE SEGURIDAD Y RELACIONES TRANSFRONTERIZAS POR PARTE DE ACTORES COLOMBIANOS EN LA TRIPLE FRONTERA AMAZÓNICA}

En este apartado se aborda la perspectiva sobre seguridad y relaciones transfronterizas por parte de los actores locales del lado colombiano de la frontera. El principal insumo para ello lo constituyen entrevistas realizadas a funcionarios y miembros de la sociedad civil $^{6}$. Teniendo en cuenta lo expuesto en el apartado teórico, el análisis está estructurado a partir de tres ejes: 1) visiones sobre el lugar de la región amazónica en el contexto nacional y global, así como la presencia estatal y la relación con el Estado central; 2) perspectivas sobre seguridad; 3) relaciones transfronterizas, enfatizando en la relación con las autoridades estatales de los otros dos países y la cooperación en seguridad.

1. En lo referente a las visiones locales sobre la región en el contexto nacional y global los participantes resaltaron la importancia de la Amazonia y su condición de territorio estratégico. Esta importancia está dada principalmente por la riqueza y diversidad medioambiental, por el río Amazonas y todas las dinámicas territoriales que se constituyen el torno a él, así como por el potencial económico que la región representa. De esta manera, uno de los participantes resalta el atractivo que constituyen las riquezas naturales y el potencial turístico para la inversión extranjera:

La importancia radica en la riqueza del río como el factor que más incide para que se originen aspectos como el comercio, transporte de personal, turismo, biodiversidad, y acceso de transporte. La región es importante porque es una zona de reserva natural, y en ella no impera la inversión nacional, sino la extranjera, para poder efectuar planes turísticos e inclusive hasta explotación mineral [...] Además, es importante por su ubicación geoestratégica, como también saber que el desarrollo y turismo está creciendo de una manera impresionante en la región (entrevista a miembro Armada Nacional).

En el mismo sentido, en otra de las entrevistas se destacan de manera particular los recursos hídricos:

La región ha despertado el interés por parte del Estado, por lo que contiene y significa la frontera, y porque este es el departamento más grande de todo el país. Tanto Colombia, Brasil y Perú son conscientes de la riqueza del agua, el agua es la verdadera riqueza, más que el petróleo [...] Además del agua, se concibe como riqueza lo cultural y lo humano, como también los minerales y la riqueza del subsuelo colombiano (entrevista a representante de la Iglesia católica).

\footnotetext{
6 Se realizaron trece entrevistas con funcionarios de la Alcaldía de Leticia, Gobernación del Amazonas, Policía, Armada Nacional, Capitanía de Puerto, Defensoría del Pueblo y representantes de diversas organizaciones en la ciudad de Leticia. Las entrevistas fueron realizadas por integrantes del Grupo de Investigación en Seguridad y Defensa (GISDE) con apoyo de la Universidad Nacional de Colombia, Sede Leticia.
} 
Un aspecto interesante es que ese reconocimiento de la importancia de la Amazonia ha estado apalancado por los actores internacionales, quienes ya sea por el interés de conservación ambiental o por el de explotación de los recursos han contribuido a posicionarla como estratégica. En palabras de uno de los participantes, "la Amazonia se vende sola" (entrevista a exfuncionario de la Defensoría del Pueblo).

Este posicionamiento internacional ha contribuido a una mayor atención por parte del Estado colombiano:

El Gobierno le ha tenido que prestar mayor atención a los antiguos territorios nacionales entre los que está el Amazonas, por la misma importancia que el Amazonas representa al mundo. Hoy el Amazonas es un sitio seguro y tranquilo, con mucha diversidad ecológica, un ambiente agradable, un río caudaloso y comunidades indígenas enfrascadas en su cultura. Colombia se ha dado cuenta que, si no le presta la atención pertinente, el mundo está atrás de él (entrevista a funcionario de la Gobernación del Amazonas).

Sin embargo, algunos actores locales perciben que el Estado ha hecho uso de la visibilidad y el interés por la región como una herramienta para obtener recursos o avanzar en sus intereses en arenas internacionales; mientras tanto, esa visibilidad coexiste con la débil presencia estatal y las precarias condiciones socioeconómicas de sus habitantes.

El departamento a nivel internacional es "una maravilla”, y que el país vende la Amazonia en el buen sentido de la palabra, para que ingresen recursos al Ministerio del Medio Ambiente. No obstante, de este monto no se le invierte a la región como debería hacerse. Si fuese así no existirían situaciones como la ausencia de servicios públicos, de agua potable siendo esta un derecho humano, de infraestructura vial, de malla vial, de acueducto ni de alcantarillado. Aunque en energía ha mejorado la región, esto se desplaza con la deficiente recolección de residuos sólidos y la contaminación del medio ambiente por falta de herramientas, cuando se supone que en esta región se debería controlar mucho más (entrevista a funcionario de la Cámara de Comercio).

Desde la perspectiva local, el discurso sobre la región tanto a nivel estatal como internacional se centra en el tema medioambiental, pero las problemáticas de interés regional no se limitan a este asunto; los pobladores locales y la complejidad del territorio exceden esta agenda y cuestionan que la acción del Estado se enfoque en la conservación, sin atender las demandas de los pobladores y la necesidad de generar fuentes de desarrollo económico e integración territorial.

La región tiene una importancia ecológica, pero necesariamente una de las políticas que debe implementar el Estado es de cómo conservar y al mismo tiempo llevarles un desarrollo sostenible a las personas. Es decir, implementar una política de empleo a través de las pequeñas industrias, con el fin de que la gente no vuelva al campo a devastar la selva, a afectar nuestro medio ambiente y a depender de esta gran biodiversidad (entrevista a exgobernador del Amazonas).

En el mismo sentido de lo expresado por los actores entrevistados, Zárate (2015) señala que la idea de una ausencia del Estado en estos territorios no es cierta, en cambio, lo que hay es "una total incoherencia, inoperancia y ausencia de unidad de acción” (p. 85) por 
parte de este, fruto, entre otros factores, de un proceso inacabado de construcción estatal y de la falta de integración de la región al modelo de desarrollo y a los espacios de decisión de política pública. Esa condición de marginalidad y aislamiento coincide con el interés por el manejo, el control y la explotación de los recursos naturales existentes en este territorio. En efecto, la explotación económica por parte de los Estados, a partir de una visión extractivista, constituyó el motor de su presencia en estos territorios (PNUMA у OTCA, 2009).

En cuanto a la presencia estatal, se resalta su precariedad y la falta de políticas públicas que atiendan a las necesidades de las poblaciones (Hurtado y Aponte, 2017). La falta de recursos, las dificultades geográficas, y el desinterés de las élites nacionales por el territorio fueron señalados como algunas de las causas de la debilidad estatal. No obstante, también se identifica un patrón de centralización de la institucionalidad en Leticia que contrasta con la ausencia del Estado en la mayor parte del departamento del Amazonas, es decir, una relación de centro-periferia:

La presencia estatal y la oferta institucional en Leticia es "buena”, pero en los nueve corregimientos y en las más de cien comunidades indígenas es diferente [...] Hay insuficiencias en agua potable, saneamiento básico y salud. [...] Aunque hay presencia estatal, hace falta mayor oferta institucional, que no solo se enfoque en Leticia (entrevista funcionario de la Cámara de Comercio).

Un aspecto importante de la presencia del Estado es que la mayoría está constituida por la Policía y las Fuerzas Militares. La debilidad de las demás instituciones del Estado no permite dar respuestas adecuadas a las problemáticas locales.

En la región escasean las instituciones de seguridad. La seguridad del departamento no radica en la capacidad de los uniformados, sino en la falta de institucionalidad en las fronteras. En Puerto Nariño se genera inseguridad y violencia permitiendo autoridades corruptas, motivadas por el empresario que trata de sobornar para no perder sus mercancías porque son ilegales, o para no perder tiempo de ir desde Puerto Nariño hasta Leticia. La principal problemática es la corrupción en las autoridades por insuficiencia institucional aduanera, no militar. En todo el departamento está la Policía y Fuerzas Militares, hasta en Encanto, Alegría, San Rafael está la Fuerza Naval del Sur y está el Batallón 50; en la zona de frontera con Brasil también está el Ejército, en Chorreras hay Policía y Ejército (entrevista a funcionario de la Cámara de Comercio).

Zárate (2015) señala que la opción que tomó el Estado colombiano para intentar administrar y ocupar el territorio amazónico desde comienzos del siglo xx fue la "colonización militar". "Esta fue la estrategia para establecer en Leticia y la frontera a policías y soldados como colonos, y llegó a convertirse en un factor predominante dentro del esquema de organización de la administración pública en toda la región amazónica colombiana” (p. 80). Si bien de manera posterior se han implementado diversidad de políticas públicas sectoriales, estas han terminado subordinadas a las políticas de defensa de la soberanía y la seguridad, y en últimas no han logrado mejorar las condiciones de vida de los habitantes de manera sustancial.

A propósito de las políticas públicas, encontramos dos perspectivas. Por un lado, al- 
gunos actores señalan que si bien hay políticas públicas que en su formulación son adecuadas y responden a las problemáticas del territorio, hay un serio problema en la implementación. Por otra parte, otros actores consideran que hay una desconexión entre el Estado central y la región. De manera que las políticas son formuladas en un "escritorio" en Bogotá, pero desconocen el contexto local, como señaló un funcionario de la Alcaldía de Leticia. En este sentido, las problemáticas identificadas por los entrevistados fueron: educación, carencia en el acceso a servicios públicos -sobre todo agua potable y alcantarillado-, falta de oportunidades de empleo, falta de regulación en el comercio fronterizo y contrabando, falta de infraestructura y aislamiento del resto del territorio nacional.

2. En la agenda de seguridad, los participantes señalaron tres tipos de problemáticas: primero, las condiciones de pobreza y precariedad en las que viven los pobladores de la región; segundo, el narcotráfico, el crimen organizado y la presencia de actores armados ilegales; tercero, las problemáticas de convivencia y seguridad ciudadana en el territorio urbano. Es interesante señalar que la seguridad ambiental no fue señalada de manera particular por las personas entrevistadas. Sin embargo, Leticia y Tabatinga presentan problemas de contaminación, sobre todo en sus quebradas, lo cual está asociado a la falta de una adecuada planeación urbana.

La condición de frontera atraviesa la relación del territorio y las dinámicas de seguridad. Desde el lado colombiano, Leticia es considerada una ciudad segura, debido a la fuerte presencia estatal. Sin embargo, no ocu- rre lo mismo con los municipios fronterizos de Brasil y de Perú. Esas zonas grises de la presencia estatal generan dinámicas particulares en la gobernanza de la seguridad en esta zona.

En materia de seguridad, gracias a la presencia de la Policía, la militar y la del sistema judicial colombiano, Leticia tiene un ambiente seguro. Sin embargo, las problemáticas de la región no se gestan en Leticia, sino al otro lado de la frontera: el tema del narcotráfico, ajuste de cuentas, descomposición social que hay en la región, producto de la falta de oportunidades laborales, hace que muchos jóvenes, hombres y mujeres se dediquen al tema ilícito, y promueven así los asesinatos selectivos con víctimas que en su mayoría son inocentes. Algunos de estos ilícitos son sucedidos (sic) en Colombia, pero la gran mayoría ocurre en Brasil y en Perú. Por ello, las problemáticas no obedecen a una Leticia insegura, sino a unos vecinos inseguros (entrevista a funcionario de la Gobernación del Amazonas).

El encuentro de tres soberanías y órdenes judiciales distintos hace que el área de frontera sea un espacio de opacidad, lo cual es aprovechado por los actores ilegales; estos navegan y se adaptan a las particularidades del territorio fronterizo. Tomando como ejemplo el narcotráfico, según la información recabada, narcotraficantes colombianos y peruanos son los encargados de los cultivos de coca y laboratorios de procesamiento del lado peruano de la frontera. La droga luego es transportada por el río Amazonas hacia Brasil, donde luego será distribuida para ser vendida en las ciudades de este país o embarcada para ser distribuida en Europa. Respecto a los asesinatos, un funcionario de la policía colombiana comentaba que muchos de ellos ocurrían en Tabatinga, pero 
las víctimas eran colombianas. Así lo confirmaba otro de nuestros entrevistados:

El narcotráfico genera inseguridad, por ejemplo, en Tabatinga se presentaron como 50 o 60 personas asesinadas y las autoridades a las que consulté sobre los motivos han llegado a la conclusión de que su totalidad se presenta por la situación del narcotráfico. La totalidad de los asesinatos se relacionan directa o indirectamente con el narcotráfico (entrevista a funcionario consular colombiano en Tabatinga).

Es decir, que la "tranquilidad" de Leticia no implica la ausencia de fenómenos ilícitos, sino su ajuste a las condiciones del territorio fronterizo. No obstante, el efecto de las rentas ilegales hace parte de la economía de la ciudad.

En la triple frontera hay paramilitares, guerrilleros, narcotraficantes y delincuencia común, pero no es visible. Leticia ya no es el centro de estas problemáticas, sino es el paso. Ni en Tabatinga ni en Santa Rosa hay suficiente control, lo que permite que en estos sectores se propague lo ilícito, no solo el tráfico sino también el homicidio de colombianos en la zona de frontera de estos dos países. A lo que se apunta en la región es a no volver a la bonanza del narcotráfico, por eso es por lo que en Colombia se ha controlado muy bien esta problemática, y en Leticia es muy buena la seguridad, aunque obviamente hay casos de casos. Eso sí, hay muchas secuelas del narcotráfico: mucha drogadicción y una canasta familiar muy costosa (entrevista a funcionario de la Alcaldía de Leticia).

La condición de "zona gris" de la frontera respecto a la jurisdicción estatal es uno de los aspectos fundamentales de la seguridad en área de fronteras que, a juicio de Adler (2017), aún no logra ser totalmente comprendido. Según esta autora, dicha situación lleva a que el territorio se convierta en un espacio de impunidad, ya que sus características otorgan ventajas a los actores criminales para evadir la acción del Estado. Por ejemplo, mientras que dichos actores cruzan los límites de manera legal o ilegal, los funcionarios estatales no pueden cruzar para perseguirlos.

3. Finalmente, respecto al eje de relaciones transfronterizas y de cooperación en seguridad, los actores locales reconocen la existencia de diversos mecanismos de cooperación. En general, señalaron que existe una buena relación con los pares de los países vecinos, así como reuniones de coordinación, consejos de seguridad y comisiones mixtas sobre el tema. Sin embargo, en materia de aplicación de la ley y control territorial, los funcionarios entrevistados manifestaron las limitaciones que les implica la condición de frontera. Por ejemplo,

... si se da el caso de una embarcación que está navegando sobre el Amazonas y que posiblemente está cargada con drogas, como son aguas internacionales, se empieza a hacer un control, y si estos se ubican en la orilla peruana, pues las autoridades de Colombia no pueden hacer ninguna intervención (entrevista a miembro de la Armada Nacional).

Otra problemática tiene que ver con las diferencias institucionales y de presencia estatal, así como los distintos marcos legislativos, los cuales representan un obstáculo para desarrollar acciones conjuntas de control territorial y aplicación de la ley.

La policía está ubicada geográficamente en 4 puntos sobre el río Amazonas, 2 sobre el Putumayo y 1 en Ca- 
quetá. Haciendo un paralelo, se observa que sobre el río Amazonas tiene 4 y Perú 7; Putumayo 2 y Perú 12. Este es el segundo obstáculo: la desproporción significativa entre policías de cada Estado. Incluso, las diferencias con Brasil son mucho más significativas que con las de Perú. [...] Ahora bien, ¿por qué no hay facilidad en la coordinación? En Perú los narcóticos no lo tienen la Policía sino la Dirección Nacional Antinarcóticos. Desde esta falta de coordinación, comienzan a presentarse dificultades o retos en la frontera (entrevista a miembro de la Policía Nacional).

Ni en Perú ni en Brasil hay instituciones homólogas a la Defensoría. En Brasil hay algo parecido a la Defensoría, pero con otra estructura, pues es sistema judicial es totalmente diferente. En Leticia hay 5 abogados de los cuales se les hace la asignación a las personas que no tengan capacidad de pagar un abogado. En Brasil no hay existencia de ello. Es decir, la cooperación judicial en zona de frontera es demasiado compleja, porque por un lado con Brasil no hay casi similitudes, y con Perú, exactamente en Santa Rosa, no se puede hacer nada, por la ausencia institucional peruana (entrevista a funcionario de la Defensoría del Pueblo).

\section{El encuentro de jurisdicciones nacionales} distintas también evidencia asimetrías institucionales que pueden llevar a una visión de desconfianza respecto a los países vecinos. Así, no solamente se considera fuente de amenaza a las acciones ilícitas, sino también al tipo de relación entre los actores estatales del país vecino con los actores ilegales.

Entre Leticia y Tabatinga la relación de cooperación local es excelente, se ha visto la cooperación policial entre Colombia y Brasil en cuanto a la búsqueda de delincuentes. Pero se observa permisividad de las autoridades peruanas con el tema del control del narcotráfico, sobre todo porque es evidente la presencia de narcotraficantes en Santa Rosa y sus cultivos ilícitos [...] En la frontera de Perú (dicen las fuerzas militares con su supuesta evidencia de fotos satelitales) existen extensos cultivos de droga y una amplia red de narcotraficantes, que generan el narcoterrorismo, permitido por la indulgencia de las autoridades peruanas (entrevista a funcionario de la Capitanía de Puerto).

La cooperación transfronteriza en seguridad a nivel local es obstaculizada por el centralismo y la falta de implementación de los acuerdos firmados entre los tres países. En este sentido, existen canales de comunicación y coordinación entre los actores locales, pero carecen de herramientas para iniciativas conjuntas o la toma de decisiones rápidas, ya que la mayoría de asuntos debe pasar primero por los ministerios de Relaciones Exteriores de los respectivos países.

Las entidades pueden tener mucho interés en hablar entre prefecto y alcalde, pero ese no es el canal para la cooperación bilateral. El canal es entre las Cancillerías de cada parte. Lo que se requiere es mayor presión en Bogotá entre los gobernantes y congresistas de la región, para que estos convenios se den y se apliquen. Hasta el momento no hay resultados de los congresistas. El problema en cuanto a cooperación no radica en si hay convenios o no, sino en la efectividad que estos deberían tener (entrevista a funcionario de la Defensoría del Pueblo).

No obstante, los funcionarios locales han desarrollado formas de cooperación informal para hacer frente a las problemáticas de seguridad sin tener que pasar por los Estados 
centrales y en ausencia de canales descentralizados formales:

En materia de cooperación no es clara. La policía extralegalmente hace convenios, pero eso es cooperación interna informal. Es decir, se requiere algo informal para acabar con la impunidad, y no debería ser así, pero en lo cotidiano es tanto normal como lo mejor para hacer justicia. La frontera es sui generis en la cotidianidad, pero a nivel de entidades estatales es muy complejo, porque los trámites tienen que hacerse por medio de Cancillería (entrevista a funcionario de la Alcaldía de Leticia).

\section{CONCLUSIONES}

Las críticas a la literatura tradicional sobre fronteras en ciencia política y relaciones internacionales, así como el debate respecto al proceso de política pública y el territorio han puesto de presente la necesidad de analizar la seguridad teniendo en cuenta las particularidades de las áreas de frontera. En este contexto, el objetivo central del artículo ha sido describir $\mathrm{y}$ analizar las visiones sobre seguridad y relaciones transfronterizas por parte de actores locales en el lado colombiano de la triple frontera amazónica Brasil-Colombia-Perú y, por esta vía, contribuir al estudio de un territorio que hasta ahora sigue siendo poco comprendido en los estudios de seguridad y defensa.

El análisis ha permitido constatar que el carácter fronterizo imprime particularidades a las dinámicas de seguridad y cooperación. La lógica centro-periferia atraviesa la relación de los Estados con estos territorios, en este sentido, desde la perspectiva local resaltan dos aspectos: 1) la precariedad estatal y la falta de políticas públicas efectivas que brinden condiciones de vida digna para sus habitantes; 2) el interés en la región por parte de actores estatales y no estatales debido a la potencialidad de explotación de recursos naturales, así como de servicios, en especial el turismo. Por otra parte, la fuerte presencia institucional en la ciudad de Leticia también ha configurado una relación de centro-periferia vis-á-vis los territorios circundantes.

En cuanto a la agenda de seguridad, las condiciones de pobreza y marginalidad de los pobladores, el narcotráfico, el crimen organizado y la presencia de actores ilegales constituyen las principales problemáticas señaladas por los participantes. Un aspecto interesante es que la seguridad ambiental, que es un asunto central en los discursos internacionales sobre la Amazonia, no fue identificada como un asunto de especial relevancia.

La condición de frontera moldea los patrones de criminalidad y violencia, de manera que los actores ilegales navegan en medio de las diferentes jurisdicciones nacionales y aprovechan las "zonas grises" para evitar la persecución por parte del Estado. Es así como en este territorio, que constituye una zona gris de la soberanía estatal, confluyen las prácticas de los Estados con las de los actores ilegales y los pobladores, así como de instituciones y mercados formales, informales e ilegales. En este contexto, la gobernanza de la seguridad no se ajusta a un modelo tradicional de soberanía estatal, ya que mientras los funcionarios estatales tienen como límite la frontera nacional, los habitantes y los actores criminales transitan fluidamente a través del territorio conformado por el espacio fronterizo entre 
estos tres países. De manera que un aspecto que afecta las relaciones entre los Estados y las políticas frente a las amenazas transnacionales son las asimetrías, ya sean de tipo económico o institucional. En ciertos casos, estas generan incentivos para que las actividades ilícitas sean toleradas, bien sea por actores estatales nacionales o locales.

En el ámbito institucional, las diferencias entre la capacidad estatal para la implementación de políticas de seguridad obstaculizan la generación de estrategias conjuntas de gobernanza y dan origen a percepciones de desconfianza por parte de los actores estatales colombianos respecto a sus pares en los países vecinos, al considerar que toleran o cooperan con los actores ilegales. Otro punto clave en las relaciones transfronterizas entre actores estatales tiene que ver con los canales formales de cooperación en seguridad, los cuales usualmente se dan a nivel central entre las capitales sin tener en cuenta a los territorios. Esta desconexión afecta la acción conjunta por parte de los funcionarios locales, quienes frente a los problemas y desafíos a los que deben hacer frente han desarrollado formas de cooperación informal.

\section{REFERENCIAS}

Aguiar, J. (2010). Strething the border: Smuggling practices and the control of illegality in South America. New Voices Series 6, Global Consortium on Security Transformation.

Andreas, P. (2003). Redrawing the line: Borders and security in the twenty-first century. International Security, 28 (2), 78-111.
Aponte Motta, J. (2007). Estado nación e identificación en la Amazonia. Múltiples fronteras, el vértice oriental del Trapecio Amazónico. Ponencia presentada en el Congreso Europeo de Americanistas CEISAL, Université livre de Bruxells.

Aponte Motta, J. (2011). La frontera en el espacio urbano: expresiones del límite entre Leticia (Colombia) y Trabatinga (Brasil). Mundo Amazónico (2), 199-223.

Aponte Motta, J. (2016). Leticia y Tabatinga. ¿¿Pequeñas ciudades fronterizas amazónicas? Mundo Urbano, 47.

Aragón, L. (2007). Novos temas regionais para o estudo da Amazônia. En S. Feldman y A. Fernandes (orgs.). O urbano e o regional no Brasil contemporâneo: Mutaçôes, tensöes, desafios. Salvador, Bahia: ANPUR/UFBA.

Bartolomé, M. (2005). Antropología de las fronteras en América Latina. Ameriquest, 2 (1).

Becker, B. (2005). Geopolítica da Amazônia. Estudos Avançados, 19 (53).

Betancourt Vélez, R. y Pachón, Ó. S. (2013). otca: el Amazonas en el horizonte de la política exterior colombiana. Papel Politico, 18 (1), 343-365.

Bitencourt, L. (2002). The importance of the Amazon Basin in Brazil's evolving security agenda. En J. Tulchin y H. Golding (eds.). Environment and Security in the Amazon Basin. Washington: Woodrow Wilson Center.

Boisier, S. (2009). El Norte Grande de Chile y sus dos Triple-Fronteras: Andina (Perú, Bolivia y Chile) y Circumpuneña (Bolivia, Argentina y Chile). Cuadernos interculturales, 7 (13), pp. 27-42.

Casar, M. y Maldonado, C. (2008). Formación de agenda y procesos de toma de decisiones: una aproximación desde la ciencia política. Documento de Trabajo 207. México D.F.: CIDE. 
Comisión Nacional para el Desarrollo y Vida sin Drogas - Devida (2018). Desarrollo en el Trapecio Amazónico. Recuperado de http://www.devida. gob.pe/desarrollo-en-el-trapecio-amazonico/

Departamento Nacional de Planeación (DNP) (2014). CONPES 3805 de 2014 - Prosperidad para las fronteras de Colombia. Recuperado de https:// www.dnp.gov.co/programas/desarrollo-territorial/Ordenamiento $\% 20 y \% 20$ Desarrollo $\% 20$ Territorial/Paginas/implementacion-de-la-politica-de-fronteras.aspx

Gallo, N. (2015). Fronteras en Colombia: ¿estratégicas, amenaza u oportunidad para la seguridad y defensa? Washington: William J. Perry Center for Hemispheric Defense Studies. Recuperado de http://chds.dodlive.mil/files/2015/08/pub-ppcampos.pdf

Grimson, A. (2004). Fronteras, naciones y región. Ponencia presentada en el Foro Social de las Américas, Quito.

Grisales, G. (2005). ¿Amerita la frontera de Colombia, Brasil y Perú una zona de integración trinacional? Aldea Mundo, 10 (18), pp. 54-61.

Guedes da Costa, T. (2002). sIvam: Challenges to the effectiveness of Brazil's monitoring project for the Amazon. En J. Tulchin y H. Golding (eds.). Enviroment and Security in the Amazon Basin. Washington: Woodrow Wilson Center.

Hurtado, A. y Aponte, J. (2017). ¿Hacia un gobierno transfronterizo? Explorando la institucionalidad para la "integración” colombo-peruana. Estudios Fronterizos, 18 (35), 70-89.

Idler, A. (2018). Preventing conflict upstream: Impunity and illicit governance across Colombia's borders. Defence Studies, 18 (1), 58-75.

Londoño, E. (2015). Política externa para la integración fronteriza a partir del caso de la Triple Frontera Brasil, Colombia y Perú en los gobiernos de
Lula, Uribe y García (2006-2011). Análisis Político, 83, 44-56.

López, N. y Tuesta, D. (2015). Economías ilícitas y orden social: la frontera de Perú, Brasil y Colombia. Revista CIDOB, 111, 79-104.

Machado, L. (2000). Limites e fronteiras: Da alta diplomacia aos circuitos da ilegalidade. Revista Territorio, 8, 9-29.

Machado, L., Reyes Novaes, A. y Rego Monteiro, L. (2009). Building walls, breaking barriers: Territory, integration and the rule o law in frontier zones. Journal of Borderlands Studies, 24 (3).

Mariscal, N. (2003). Teorias politicas de la integración europea. Madrid: Tecnos.

Martins Filho, J. y Zirker, D. (2000). Nationalism, national security, and Amazônia: Military perceptions and attitudes in contemporary Brazil. Armed Forces \& Society, 27 (1), 105-129.

Navarrete Ulloa, C. A. (2016). Institucionalismo informal: tras las huellas de un discurso institucionalista informal. Perfiles latinoamericanos, 24 (47), 283-306.

Newman, D. (2003). On borders and power: A theoretical framework. Borderlands Studies, 18 (1).

Newman, D. (2006). The lines that continue to separate us: Borders in our 'borderless' world. Progress in Human Geography, 30 (2), 143-161.

Oficina de las Naciones Unidas contra la Droga y el Delito unOdC (2017). Perú: monitoreo de cultivos de coca 2016. Lima: UNODC y DEVIDA.

Pabón, N. (2012). Inseguridad y perspectivas de cooperación en la región amazónica. En A. Vargas. El prisma de las seguridades en América Latina: escenarios regionales y locales. Buenos Aires: CLACso. Palacio, G. (2007). Cinco ejes analíticos para comprender la Amazonía actual. Bogotá: Friedrich-EbertStiftung en Colombia (FesCOL). 
Programa de las Naciones Unidas para el Medio Ambiente (pnuma) (2009). Perspectivas del Medio Ambiente en la Amazonía: GEO Amazonía. Panamá: PNUMA y оTCA.

Simmons, C. S., Caldas, M. M., Aldrich, S. P., Walker, R. T., Perz, S. G. y Perz, S. G. (2007). Spatial processes in scalar context: Development and security in the Brazilian Amazon. Journal of Latin American Geography, 6 (1), 125-148.

Tapia, M. (2012). Frontera y migración en el norte a partir del análisis de los censos población. Siglos XIX-XXI. Revista de Geografia Norte Grande, 53, 177-198.

Trejos, L. (2015). (2015). El lado colombiano de la frontera colombo-brasilera: una aproximación desde la categoría de área sin ley. Estudios fronterizos, 16 (31), 39-64.

Vargas, A. (2010). Inseguridad en la región amazónica: contexto, amenazas y perspectivas. Bogotá: Universidad Nacional de Colombia.

Vargas, J. P. (2011). Territorialización de las políticas públicas. En Territorialización de políticas públicas. San José: Fundación Demuca.

Vergel, F. (2008). Ciudades gemelas en fronteras amazónicas: estudio de caso Leticia y Tabatinga. Cuadernos de Vivienda y Urbanismo, 1 (2), 348-393.
Veyrunes, E. (2008). Las amenazas percibidas para la Amazonia: un estado del arte en términos de seguridad ambiental. Documento de Investigación 28, Centro de Estudios Políticos e Internacionales (CEPI). Bogotá: Editorial Universidad del Rosario.

Wilson, T. y Donnan, H. (1998). Border Identities: Nation and State at International Frontiers. Cambridge: Cambridge University Press.

Zárate, C. G. (2015). Estado, militares y conflicto en la frontera amazónica colombiana: referentes históricos para la interpretación regional del conflicto. Mundo Amazónico, 6 (1), 73-96.

Zárate, C. G. (2017). Estado, conflitos y violência en la frontera Amazónica de Brasil, Colombia y Perú. Revista Paz y Conflictos, 10 (1), 113-136.

Zárate, C. G. y Aponte, J. (2016). Leticia, Tabatinga, Santa Rosa. Pasado, presente y retos de las ciudades pares en la Amazonía. Presentación Simposio Internacional - Derecho a la Ciudad. Bogotá, 24 y 25 de agosto.

Zartman, W. (2010). Understanding life in the borderlands. Boundaries in depth and notion. Athens: University of Georgia Press. 